\title{
A CAD System for Quantifying COPD Based on 3-D CT Images
}

\author{
Jiro Nagao ${ }^{1}$, Takahisa Aiguchi ${ }^{1}$, Kensaku Mori ${ }^{1}$, Yasuhito Suenaga ${ }^{1}$, \\ Jun-ichiro Toriwaki ${ }^{2}$, Masaki Mori ${ }^{3}$, and Hiroshi Natori ${ }^{4}$ \\ 1 Graduate School of Information Science, Nagoya University, Japan \\ \{jnagao, taiguchi, mori, suenaga\}@suenaga.cse.nagoya-u.ac.jp \\ 2 School of Computer and Cognitive Sciences, Chukyo University, Japan \\ jtoriwak@sccs . chukyo-u.ac.jp \\ 3 Sapporo Kosei Hospital, Japan, TAE03517@nifty.ne.jp \\ 4 Department of Diagnostic Ultrasound and Medical Electronics, \\ Sapporo Medical University, Japan, hnatori@sapmed.ac.jp
}

\begin{abstract}
This paper proposes methods for automated extraction of emphysematous lesions from three-dimensional (3-D) CT images and quantitative evaluation of their distribution pattern. We employ 3-D image processing techniques on computer-aided diagnosis of pulmonary emphysema. Emphysematous lesions are automatically extracted by a region growing method. To analyze spatial distribution of the detected low-attenuation areas, we examine the relations between emphysematous lesions and bronchi or pulmonary blood vessels using Euclidean distance transformation. Experimental results show that the methods could be used to extract emphysematous lesions appropriately and could quantitatively evaluate their distribution pattern.
\end{abstract}

\section{Introduction}

Pulmonary emphysema is defined as "a condition of the lung characterized by abnormal, permanent enlargement of the air spaces distal to the terminal bronchiole, accompanied by destruction of their walls," which prevents a patient from proper breathing. Along with chronic bronchitis, it forms Chronic Obstructive Pulmonary Disease (COPD), which was the fifth most common cause of death in the world in the year 2002. Since the disease involves destruction of alveoli, early diagnosis, treatment and effective prevention are essential.

Pulmonary emphysema is diagnosed by physiological indices such as pulmonary function tests. In addition, image-based diagnosis of chest X-ray and CT images has recently been introduced. Generally, medical doctors visually inspect CT images for Low-Attenuation Areas (LAA). For more objective diagnosis, however, computer-aided diagnosis is required.

Earlier studies of image-based diagnosis of emphysema based on chest X-ray and CT images were aimed at quantifying the severity of the disease. Most of these diagnosis methods extract lesions as LAAs by a thresholding operation and evaluate their severity by the percentage of the LAAs (LAA\%) in the lung. The 
LAA\% is reported to be practical through comparisons with doctors' assessments and physiological indices [1]. Reference [2] showed that an index that quantifies connectedness of LAAs is a good measure, even in fibrosis cases, which raise all the $\mathrm{CT}$ values in the lungs. Reference [3] examined the difference between distributions of LAAs in the upper and lower lung regions and its effect on the lung function.

All studies mentioned above process X-ray CT slices two-dimensionally, and none of them use 3-D image processing techniques. Three dimensional processing of X-ray CT images provides information on lesions such as their 3-D distribution. In this paper, we propose methods to automatically extract emphysematous lesions by $3-\mathrm{D}$ image processing techniques and quantitatively evaluate the $3-\mathrm{D}$ distribution pattern of the extracted lesions in the lungs. Section 2 describes the proposed methods for lesion extraction and quantitative analysis. Experimental results are discussed in Sect. 3.

\section{Lesion Extraction and Distribution Analysis}

The process consists of three components: (a) preprocessing, (b) lesion extraction by a region growing method, and (c) distribution analysis based on 3-D distance.

\subsection{Preprocessing}

For preprocessing, we perform intensity (CT value) correction of CT images, slice interpolation, and extraction of the lung region, bronchi, and pulmonary blood vessels. Because of the influence of gravity on blood flow in the lung, CT images taken in the supine position show higher $\mathrm{CT}$ values on the posterior side and lower on the anterior side. This affects lesion extraction based on intensity, and hence it causes under- and overextraction of lesions. We correct this nonuniformity by lowering intensity values on the posterior side and raising it on the anterior side using the method described in [2]. Slice interpolation is employed for isotropic distance, since it is necessary in the distance transformation we will later use. In addition, we extract the lung region by thresholding to limit processing areas. Bronchial and pulmonary vascular regions are also segmented for the use of distribution analysis described later. Hereafter, these extracted bronchial and pulmonary vascular regions are abbreviated to BV regions.

\subsection{Lesion Extraction}

Emphysematous lesions are observed as LAAs, surrounded by normal, highintensity areas in CT images. Therefore, lesions are extracted by detecting lowintensity and low-variance areas. The extraction algorithm utilizes a region growing method. The growing process begins from certain starting points and grows the region to include neighboring points under the conditions described below [5].

Voxels whose intensity values are less than or equal to the threshold value $t_{\mathrm{a}}$ are included in the growing region. If the intensity of a voxel is higher than $t_{\mathrm{a}}$ 
and within the range $t_{\mathrm{a}}$ to $t_{\mathrm{b}}$, it is possible that the voxel belongs to a lesion. Therefore, we include it into the region if its intensity falls within the pre-defined range and only if the variance of the intensities in the neighborhood region is low. The acceptable limit of the variance is set high, negatively proportionally to the mean in the neighborhood, ranging from $d$ to $d+c$. The above growing conditions are formulated by:

Condition A: $\quad \mathrm{E}^{[6]}\left(\mathrm{p}_{i}\right)<a$,

Condition B: $\left\{a \leq \mathrm{E}^{[6]}\left(\mathrm{p}_{i}\right)<b\right\} \wedge\left\{\mathrm{V}^{[6]}\left(\mathrm{p}_{i}\right)<c \frac{\mathrm{E}^{[6]}\left(\mathrm{p}_{i}\right)-a}{b-a}+d\right\}$,

where $\mathrm{E}^{[6]}\left(\mathrm{p}_{i}\right)$ and $\mathrm{V}^{[6]}\left(\mathrm{p}_{i}\right)$ are the mean and variance of the intensities in the 6 neighbors of a voxel $\mathrm{p}_{i}$, respectively. The variable $i$ is the index that shows each voxel in the lung region. The constants $a, b, c$, and $d$ are adjusted manually. We select the voxels whose intensity values are lower than $s=\frac{a+b}{2}$ as the starting points of the growing process.

\subsection{Distribution Analysis}

The proposed CAD system quantitatively analyzes the distribution pattern of the lesions in three ways:

1. Lesion distribution pattern in the BV-neighborhood regions

2. Lesion concentration

3. Relations between distance from the hilar region and lesions and BV regions

Items 1 and 2 are quantified by measuring the distance between lesions or normal structures and the BV regions, and the distance between lesions and normal structures, respectively. These distances are obtained by 3-D Euclidean distance transformation. The concentration quantifies denseness of lesions. In the third item, we analyze the variations in the distribution patterns of lesions and BV regions in each lung part divided by the method described in the later section.

\section{Lesion Distribution in the Neighborhood Regions of Bronchi and Pul-}

monary Blood Vessels. Here, we quantitatively evaluate the distribution of lesions by counting the number of voxels classified into the BV-neighborhood region $R_{\mathrm{bv}}$. The region $R_{\mathrm{bv}}$ is defined as the region located within a distance $e$ from the BV regions and formulated as:

$$
\begin{gathered}
R_{\mathrm{bv}}=\left\{\mathrm{p}_{i} \mid \mathrm{D}_{\mathrm{bv}}\left(\mathrm{p}_{i}\right) \leq e\right\}, \\
\mathrm{D}_{\mathrm{bv}}\left(\mathrm{p}_{i}\right)=\min \left\{\mathrm{D}\left(\mathrm{p}_{i}, \mathrm{bv}_{j}\right)\right\} \quad j=1, \cdots, K,
\end{gathered}
$$

where $\mathrm{D}\left(\mathrm{p}_{i}, \mathrm{bv}_{j}\right)$ represents the Euclidean distance between a voxel $\mathrm{p}_{i}$ in the lung and a voxel $\mathrm{bv}_{j}$ in the BV regions, and $K$ is the number of pixels in the BV regions. Changing the distance $e$, the system investigates the differences of the lesion distribution pattern between regions near and far from the BV regions. 
Lesion Concentration. Concentration of lesions is quantified by measuring $\mathrm{D}_{\nu}\left(\lambda_{i}\right)$, the distance between a lesion voxel $\lambda_{i}$ and the nearest voxel of the normal structure. If the rate of lesions located far from the normal structure is high, the lesions can be assumed to be concentrated. In this sense, we call such cases "concentrated". Here, normal regions are defined as those that are not classified as lesion regions. We categorize lesion voxels into three types based on the distance from the normal structure: Outer, Medium, and Inner types. We then calculate the rate of the number of lesion voxels belonging to each category over the total number of the lesion voxels in the lung region.

A lesion voxel $\lambda_{i}$ is categorized as:

$$
\text { Lesion voxel category }= \begin{cases}\text { Outer type } & \text { if } \mathrm{D}_{\nu}\left(\lambda_{i}\right) l, \\ \text { Medium type } & \text { if } l \leq \mathrm{D}_{\nu}\left(\lambda_{i}\right)<m \\ \text { Inner type } & \text { otherwise }\end{cases}
$$

where

$$
\mathrm{D}_{\nu}\left(\lambda_{i}\right)=\min \left\{\mathrm{D}\left(\lambda_{i}, \nu_{j}\right)\right\} \quad j=1, \cdots, L,
$$

and $l, m$ are the constants defined beforehand, $\nu_{j}$ is a normal voxel, and $L$ is the total number of normal voxels. The lesion concentration is defined by the following equation:

$$
\text { Concentration }=\frac{\text { Inner } \%}{\text { Outer } \%},
$$

where Inner\% and Outer\% represent the rates of the voxels classified into Inner and Outer types over all of the lesion voxels, respectively.

\section{Relations between Distance from the Hilar Region and Lesions and}

BV Regions. This analysis characterizes the distributions of lesions and BV regions in the peripheral and central parts of the lung. The central and peripheral parts of the lung are defined as the parts that are near and far from the hilar region, since bronchi and blood vessels show branching patterns that start from the hilar region. In this process, we divide the lung region based on the distance from the hilar region. The numbers of lesions and BV voxels are counted for each divided region, after which rate of the counted number over the whole volume of each division is calculated.

The actual lung area division process is described as the following way. A voxel $\mathrm{p}_{i}$ in the lung region is classified into a divided region $\mathrm{R}_{j}$,

$$
\mathrm{p}_{i} \in \mathrm{R}_{j} ; \quad j=\left\lfloor\frac{\mathrm{D}_{\mathrm{h}}\left(\mathrm{p}_{i}\right)-1}{\mathrm{D}_{\text {interval }}}\right\rfloor,
$$

where $\mathrm{D}_{\text {interval }}=\frac{\max \left\{\mathrm{D}_{\mathrm{h}}\left(\mathrm{p}_{i}\right)\right\}}{N}, \mathrm{D}_{\mathrm{h}}\left(\mathrm{p}_{i}\right)$ is the distance between a voxel $\mathrm{p}_{i}$ and the hilar region, $N$ is the number of divided regions, and $\max \left\{\mathrm{D}_{\mathrm{h}}\left(\mathrm{p}_{i}\right)\right\}$ represents the maximum value of $\left\{\mathrm{D}_{\mathrm{h}}\left(\mathrm{p}_{i}\right)\right\}$. The hilar region is manually specified as a point in the pulmonary artery on the slice image where the carina is visible. 


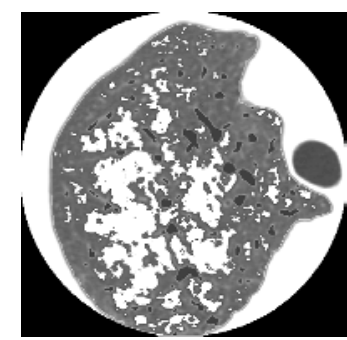

Proposed method

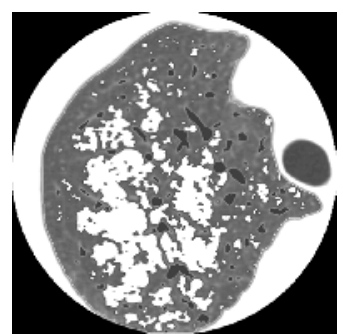

Simple thresholding operation

Fig. 1. Examples of lesions extracted by the proposed method and a simple thresholding operation (the Mod-severe case). The extracted lesions are overlaid on the original CT image as white areas.

\section{Results and Discussions}

We applied the proposed method for extracting and analyzing emphysematous lesions. Five cases, which include one normal case and four abnormal cases, were used in the experiments. The original CT images were taken by multi-detector row CT scanners $(512 \times 512$ pixels, 81 - 114 slices, $0.23-0.31-\mathrm{mm}$ pixel spacing, $0.62-\mathrm{mm}$ reconstruction pitch, $120 \mathrm{kVp}, 200 \mathrm{mAs})$. Each of the five cases was classified by a medical doctor into Normal, Mild, Moderate, Mod-severe and Severe types.

\subsection{Lesion Extraction}

Figure 1 shows the lesions extracted from the Mod-severe case. The extracted lesions are overlaid as white areas on the CT image. For comparison, the lesion regions extracted by a simple thresholding method are also shown in the same figure. The parameters for the region growing method are experimentally determined to obtain extraction results comparable to those of the doctor's. We used the same threshold value, -945 H.U., for selecting the starting points of the growing process as the threshold value for the simple thresholding operation. The ratios of the extracted lesions and of the BV regions over the whole lung region are shown in Table 1 . From the table, it is clear that the calculated rates increase in proportion to the severity determined by the medical doctor.

Although the extraction results provided by the two methods shown in Fig. 1 are very similar, closer observation reveals that the proposed method can extract areas whose intensity values are constantly low and that are surrounded by regions of higher intensity. These areas cannot be extracted by the simple thresholding method. By employing the variance of intensity values in the region growing condition, the proposed method is able to suppress the extraction of the region whose intensity values are higher than those of the surrounding region existing in the low $\mathrm{CT}$ value region. The proposed method showed better extraction results for the cases of diffuse emphysema in comparison with the 
Table 1. Rates of the extracted lesions and of the BV regions over the whole lung region $(\%)$

\begin{tabular}{|r|c|c|c|}
\hline & \multicolumn{2}{|c|}{ Emphysematous lesions } & \\
\cline { 2 - 3 } Grade & proposed method & simple thresholding & BV regions \\
\hline Normal & 0.2 & 0.2 & 7.5 \\
Mild & 6.7 & 5.0 & 6.4 \\
Moderate & 17.4 & 20.1 & 6.8 \\
Mod-severe & 17.1 & 16.9 & 5.8 \\
Severe & 50.3 & 53.0 & 4.0 \\
\hline
\end{tabular}

Table 2. Lesion distribution pattern in the BV-neighborhood regions (\%)

\begin{tabular}{|r|r|r|r|r|}
\hline & \multicolumn{4}{|c|}{ Distance $e$} \\
\cline { 2 - 5 } Grade & $1.5 \mathrm{~mm}$ & $2.2 \mathrm{~mm}$ & $2.8 \mathrm{~mm}$ & $3.4 \mathrm{~mm}$ \\
\hline Normal & 0.2 & 0.2 & 0.2 & 0.1 \\
Mild & 1.3 & 3.2 & 4.8 & 5.9 \\
Moderate & 4.2 & 8.8 & 12.1 & 14.5 \\
Mod-severe & 2.7 & 6.7 & 10.0 & 12.7 \\
Severe & 9.0 & 25.6 & 33.8 & 41.8 \\
\hline
\end{tabular}

simple thresholding method, because the proposed method considers additional information in the neighborhood such as mean intensity values and standard deviations of intensity values, which are not used by the simple thresholding method.

\subsection{Distribution Quantification}

Distribution of Lesions in the BV-neighborhood Regions. We calculated the rates of the volumes of lesions in the BV-neighborhood regions over the whole volume of the regions, changing the distance $e$ of (3). The measured rates are shown in Table 2. Compared with the rates of lesions presented in Table 1, the rates in this table are smaller for all $e$ values. The rates also increase in proportion to the distance from the BV regions. This means that the lesion rate tends to be low near bronchi or blood vessels.

Lesion Concentration. Table 3 presents the computed rates of the numbers of the lesion voxels classified into each type (Outer, Medium, and Inner) over the total number of the lesion voxels. The parameters for the categorization, $l$ and $m$ in (5), are determined so that the rates are roughly equal to each of the three types in the Severe case. The lesion concentrations calculated by (7) were 0.02, 0.04, 0.12, and 0.49 in the Mild, Moderate, Mod-severe and Severe cases, respectively. Although there were few differences between the rates of lesions in the Moderate and Mod-severe cases, as shown in Table 1, the concentration can be used to classify them. In milder cases, the lesions generally exist as spheres; however, as the disease progresses, the lesions connect with each other 
Table 3. Distance from the normal structure and the rates of lesions (\%)

\begin{tabular}{|r|ccc|c|}
\hline & \multicolumn{3}{|c|}{ Lesion type } & \\
\cline { 2 - 4 } Grade & Outer Medium Inner & Total \\
\hline Mild & 85.0 & 13.3 & 1.7 & 100 \\
\hline Moderate & 73.2 & 23.6 & 3.1 & 100 \\
\hline Mod-severe & 67.9 & 24.2 & 7.9 & 100 \\
\hline Severe & 41.8 & 37.6 & 20.6 & 100 \\
\hline
\end{tabular}

Table 4. Relation between the distance from the hilar region and the lesions in each division (\%). Bold type represents the divisions where most lesions exist

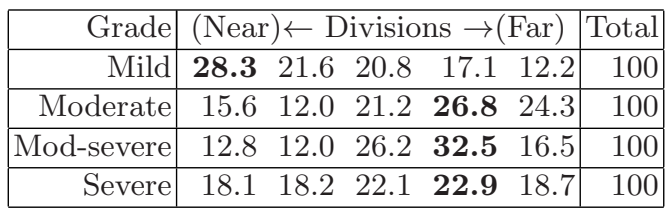

Table 5. Rates of the BV regions within each division over the corresponding division of the Normal case

\begin{tabular}{|r|r|r|r|r|r|}
\hline Grade & $($ Near $) \leftarrow$ Divisions $\rightarrow($ Far $)$ \\
\hline Normal & 100 & 100 & 100 & 100 & 100 \\
Mild & 90.0 & 73.5 & 83.1 & 99.9 & 131.9 \\
Moderate & 167.6 & 115.8 & 100.6 & 88.5 & 62.3 \\
Mod-severe & 131.4 & 71.2 & 73.1 & 80.0 & 130.8 \\
Severe & 75.3 & 55.7 & 44.2 & 34.5 & 20.2 \\
\hline
\end{tabular}

and become larger. Thus, the rates of the Inner type lesions increase. Therefore, it can be considered that the proposed lesion concentration measure can be a new index of severity of the disease, which cannot be measured by the rate of lesions over the entire lung region.

Distance between the Hilar Region and Lesions and BV Regions. From the results shown in Table 4, it is clear that lesions are mostly present at intermediate distances, i.e. between the center and the periphery in the Moderate or above cases. A study based on two-dimensional (2-D) image processing[4] showed that lesions tend to exist in the inner layer of the lung. Our analysis makes it clear that this is also true in terms of 3-D distribution.

Table 5 shows the rates of the BV regions within each divided region of the abnormal cases over the corresponding division of the Normal case. The rate of the volume of the BV regions within each division over that of the division in the Normal case was taken to be 100. The volumes of the divisions are normalized, since the volumes differ in all divisions and cases.

From this table, the rates of the extracted BV regions decrease in the Severe case, as the division increases from the hilar region. However, this does not 
hold in the cases of less severity. In these cases, the decreases of the extracted BV regions in the whole lung themselves, shown in Table 1, are not so large. Therefore, it is considered that the relation between the distance and the decrease in the volume of BV regions was not made prominent. Because the experiments were conducted on a small number of cases, further experiments on many more cases are required to investigate the supposed tendencies.

\section{Conclusion}

In this paper, we proposed a new approach to computer-aided diagnosis of pulmonary emphysema based on 3-D image processing and 3-D CT images. The 3-D region growing method was utilized for automatic extraction of lesions, which provides more intelligent and more accurate extraction than the simple thresholding method. We also proposed some feature values based on 3-D distance, which is impossible to obtain from 2-D slice processing. The experimental results revealed the following facts: (1) There are fewer lesions around bronchi and blood vessels compared to other parts of the lung. (2) The lesion concentration can be a useful measure of the disease's severity. (3) Lesions tend to exist more in the intermediate distance from the hilar region. (4) Reduction of peripheral bronchiole and pulmonary blood vessels is remarkable in the Severe case.

Future work will include further investigation into visualization of the extraction and analysis results and clarification of the relations between the proposed method and clinical knowledge.

Acknowledgments. The authors wish to thank all members of the Suenaga and Toriwaki laboratories. This study was partly supported by the Grants-inAid for Scientific Research and the 21st Century COE Program from Japan Society for the Promotion of Science, Grants-in-Aid for Cancer Research from the Ministry of Health, Labor and Welfare of Japan.

\section{References}

1. Archer, D. C., et al.: Automated in-vivo quantification of emphysema. Radiology 188-3 (1993) 835-838

2. Blechschmidt, R. A., et al.: Automated CT image evaluation of the lung: a morphology-based concept. IEEE Trans. Med. Imag. MI-20-5 (2001) 434-442

3. Saitoh, T., et al.: Lobar distribution of emphysema in computed tomographic densitometric analysis. Investigative Radiology 35-4 (2000) 235-243

4. Honma, A., et al.: Radiological and Pathological Analysis of the Distribution of Centrilobular Emphysema in the Transverse Plane of the Lung. Japanese Journal of Thoracic and Cardiovascular Surgery 33-3 (1995) 282-292

5. Mori, K., et al.: Automated Extraction of Bronchus Area from Three Dimensional X-ray CT Images. Tech. Rep. of IEICE PRU93-149 (1994) 49-56 